\title{
ANALOG VIDEO MAGNETOGRAMS IN REAL TIME
}

\author{
R. C. SMITHSON and R. B. LEIGHTON
}

California Institute of Technology, Pasadena, Calif., U.S.A.

For many years solar magnetic fields have been measured by a variety of techniques, all of which exploit the Zeeman splitting of lines in the solar spectrum. One of these techniques (Leighton, 1959) involves a photographic subtraction of two monochromatic images to produce a picture of the Sun in which the line-of-sight component of the solar magnetic field appears as various shades of gray. In a magnetogram made by this method, zero field strength appears as neutral gray, while magnetic fields of one

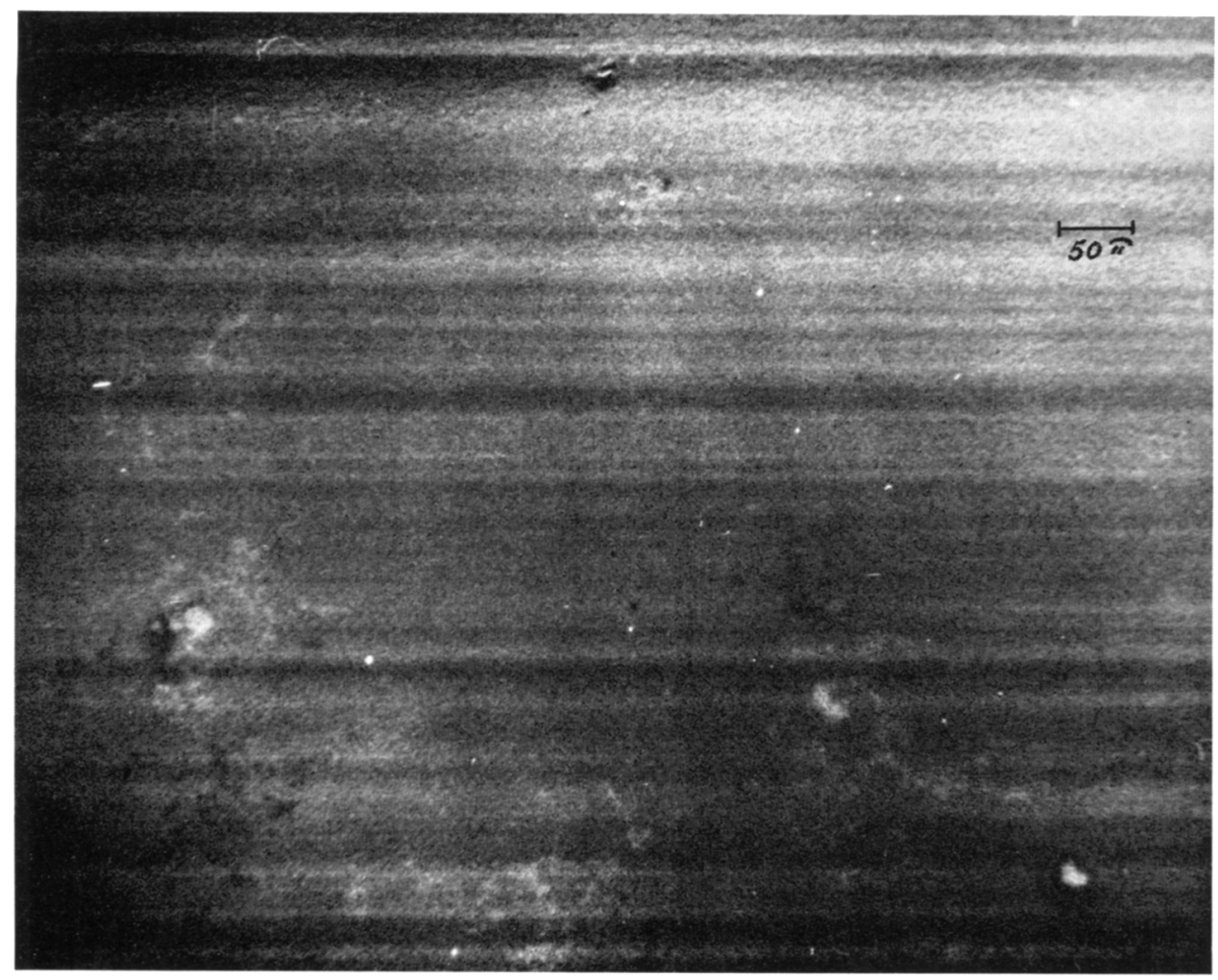

Fig. 1. An early magnetogram produced by the photographic subtraction technique. The monochromatic images were made using the spectroheliograph at the $60 \mathrm{ft}$ tower at Mount Wilson, September 11, 1960.

polarity or the other appear as lighter or darker areas, respectively. Figure 1 shows such a magnetogram.

The photographic subtraction method consists of using a beamsplitter and spectro- 
heliograph (or filter) to make two simultaneous monochromatic pictures of the Sun, one of the pictures being made in right-hand circularly polarized light and the other in left-hand circularly polarized light. Both pictures are taken in the same wavelength a wavelength that falls in the wing of a solar line susceptible to the Zeeman effect and having a simple triplet Zeeman pattern. In the presence of a magnetic field, one component of the triplet shifts above the zero-field frequency, and the other shifts an equal amount below, and these outer components are oppositely circularly polarized if the field is directed toward or away from the observer. If the frequency at which the two pictures are taken is in the wing of the absorption line where intensity is varying rapidly as a function of frequency, a small frequency shift will be translated into a difference in intensity between the two pictures (see Figure 2).

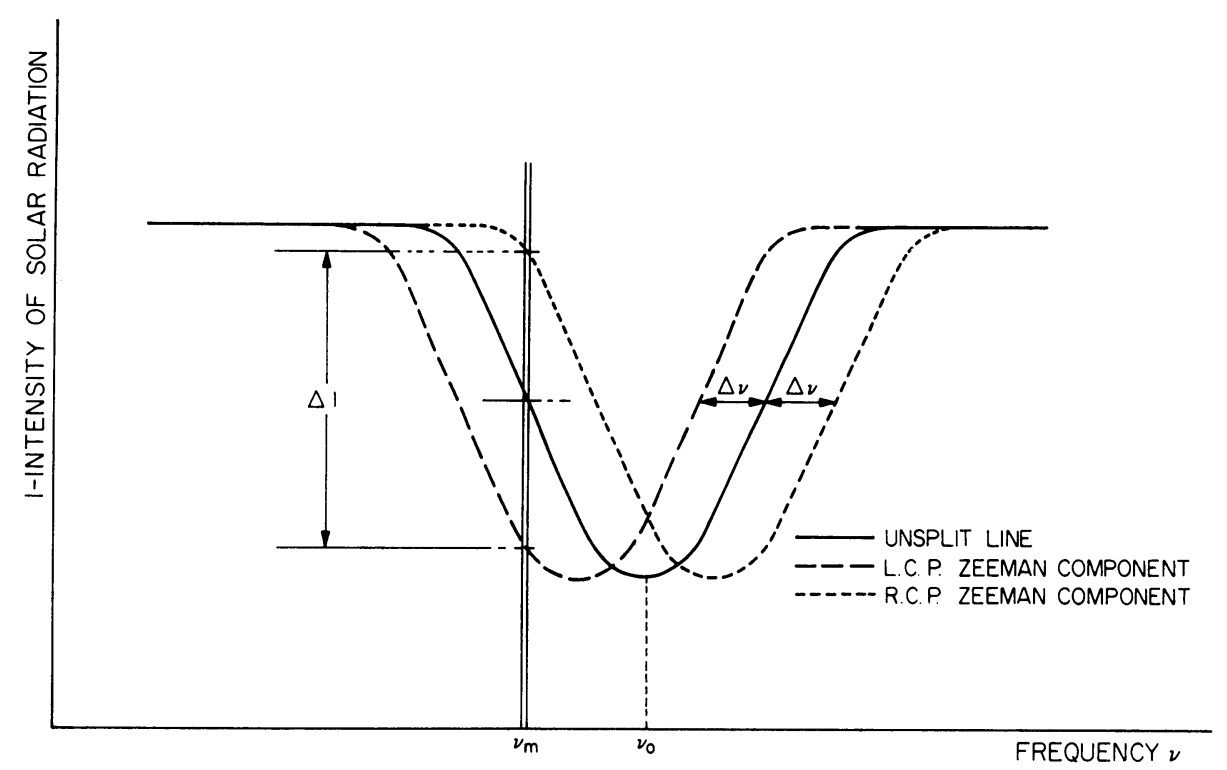

Fig. 2. The wing of the absorption line is used to translate Zeeman splitting into a difference in image intensity. $v_{0}$ is the frequency of the unsplit line, and $v_{m}$ is the frequency at which the monochromatic images are made. A shift $\Delta v$ in the frequency of the Zeeman components produces an intensity difference $\Delta I$.

Once the two pictures have been made, a unit contrast, positive copy is made of one of them in such a way that if it is superimposed upon its own negative, a uniform gray results. This positive is then superimposed upon the other negative, producing a picture in which all intensity variations common to both original pictures are cancelled, leaving only the difference between the two images as the final magnetogram (see Figure 3).

The photographic subtraction method gives magnetograms of high spatial resolution compared to those made by scanning magnetographs which use photomultiplier tubes as the light-sensing element, but is only about $1 / 10$ as sensitive in 
terms of field strength. The chief drawback of the method is the complexity of the photographic processes involved. Making suitable positive copies is an exacting and time consuming process and, typically, several days elapse before the final magnetograms are prepared. Furthermore, this complexity discourages one from preparing

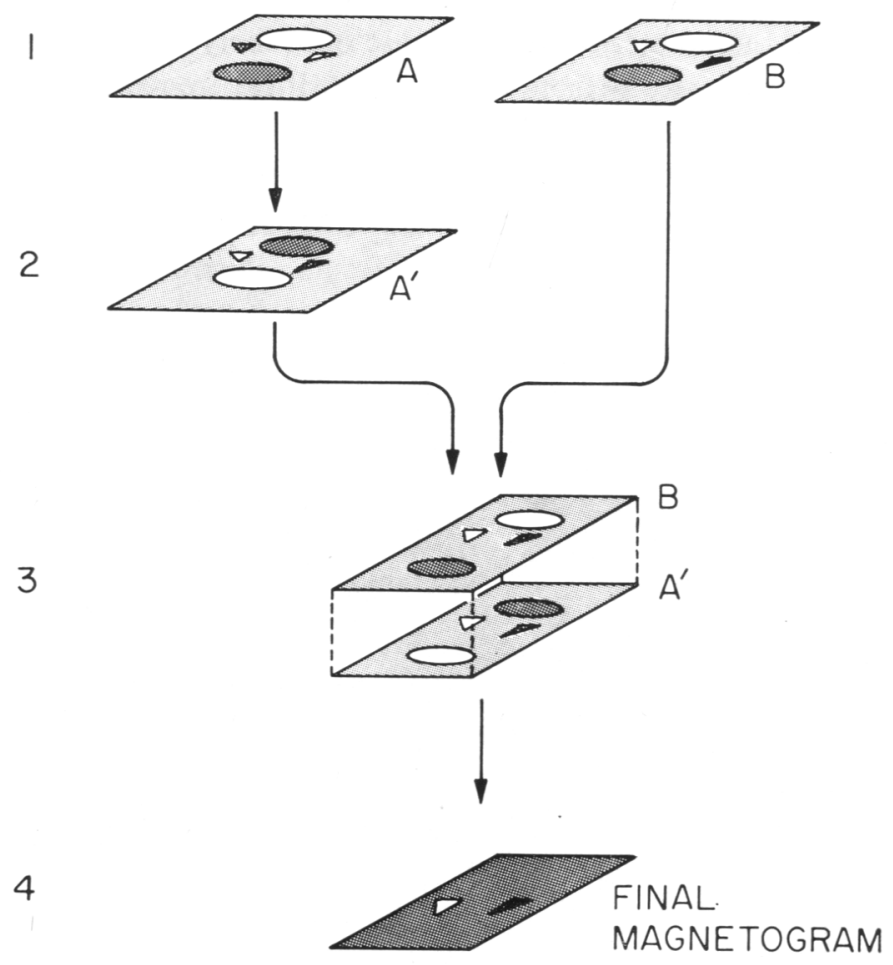

Fig. 3. The photographic subtraction method. In step 1, the two monochromatic images $A$ and $B$ have been made. In step 2, a unit contrast, positive copy of $A$ has been made ( $\left.A^{\prime}\right)$. In step $3, A^{\prime}$ and $B$ are sandwiched together to form the final magnetogram (step 4).

large numbers of magnetograms such as would be required for time-lapse movies. At Caltech, the photographic cancellation process has been automated for greater ease in processing large numbers of pictures (Roberts, 1970), but it remains timeconsuming and limited in sensitivity.

The video differential photometer described here is the result of an attempt to adapt television technology to the task of producing an electronic equivalent of the photographic subtraction technique. Advantages of electronic subtraction would be: potentially greater field sensitivity, immediate availability of the subtracted magnetograms (virtually in real time), and greater versatility in combining and comparing image data.

A definite factor in the design of the present system was the desire to keep the cost 
as low as possible. From the standpoint of cost, it seemed most desirable to use standard 525 line, 30 frame per second, commercial television equipment. Because of the large commercial market, prices are generally much lower and a much wider range of equipment is available than is available in other possible formats. Of course, a slow-scan system would have the advantages of better intrinsic signal-to-noise ratio and greater ease of digitization for computer processing of pictures. It would, however, have the serious disadvantages of higher cost and less readily available component parts. Nevertheless, it remains a promising area for future investigation.

Once the commercial format was decided upon, a signal-to-noise ratio of the camera output of about 300:1 could be assumed: this is a typical value for a moderately priced commercial television camera. Since an ability to detect intensity differences between two pictures of $1: 1000(0.1 \%)$ was considered desirable, a method of improving the signal-to-noise ratio had to be found. It was also necessary to find a means of storing the finished magnetograms.

The means used to fulfill both these functions is through a many-track video disc recorder. The system signal-to-noise ratio can then be improved by averaging several magnetograms. (The signal-to-noise ratio should be improved by a factor of nearly $N^{1 / 2}$, if $N$ magnetograms are averaged.) The recorder can also be used to store sequential pictures in right and left circularly polarized light so that the recorded pictures can be subtracted later. This eliminates the need for a beam splitter in cases when the image does not move appreciably between the times when the two pictures are recorded. A disc recorder was chosen over a video tape recorder because of its generally better signal-to-noise ratio and its much faster and easier access to individual stored pictures for subtraction or averaging. The recorder used has two movable video read/write heads with 153 tracks available to each, and two fixed video read/write heads. It is possible to read from two heads and write on a third simultaneously, a feature which is necessary for subtracting and averaging recorded pictures, and which would be almost impossible using video tape techniques.

All video subtraction, averaging, and other processing is done in analog form, since real-time digitization at commercial picture rates is difficult and storage of the digital information requires costly high-speed memory systems.

The system is under the control of a patchcord-programmable controller designed and built at Caltech. All video recording and processing, as well as control of peripheral equipment is handled automatically.

The final magnetogram is displayed on an ordinary television monitor, where it may be photographed frame-by-frame by an automatic cine camera under the control of the system automatic controller.

A functional block diagram of the differential video photometer is shown in Figure 4. The system can operate either as a two-camera system in which the two pictures to be subtracted are viewed simultaneously through a beamsplitter, or as a single-camera system in which the two pictures are recorded sequentially. In the case of the dual camera system, differences in response of the two cameras are easily averaged out by recording a (camera A-camera B) picture, then reversing the role of the two cameras 
and recording a (camera B-camera $\mathrm{A}$ ) picture. The two pictures are then averaged, retaining the desired information but cancelling out systematic differences between the two cameras.

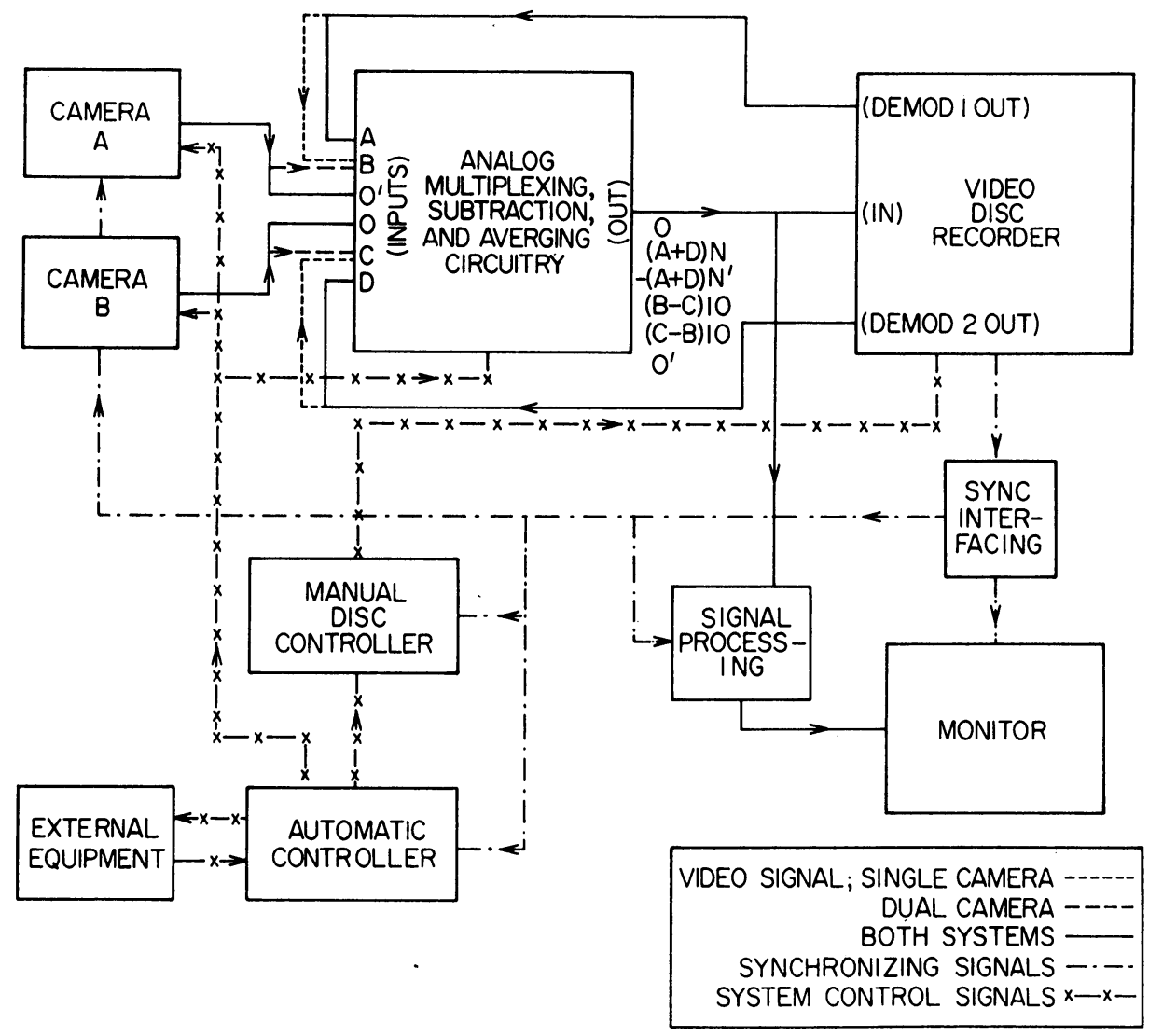

Fig. 4. A functional block diagram of the differential video photometer. Direction of flow of the various signals is indicated by arrows. A list of the outputs which may be selected is given near the output of the multiplexing circuit. The video disc recorder has two independent outputs for recorded video, labeled 'demod 1' and 'demod 2'.

In order to produce the desired $0.1 \%$ sensitivity, it is necessary to average about 100 subtracted pairs. (The actual number is taken as 128 for convenience.) An effective signal-to-noise ratio of 1000:1 can be produced by averaging, even though the disc recorder has a signal-to-noise ratio of only $100: 1$, by contrast-enhancing the subtracted pictures by a factor of about 10 , so that the voltage level of the noise to be averaged out is large compared with that of the disc recorder noise. This technique causes large difference signals to saturate, however, and sensitivities of $0.1 \%$ cannot be realized if the maximum differences to be faithfully recorded are much greater than two or three percent. Systematic differences in the recorder heads are averaged out in 


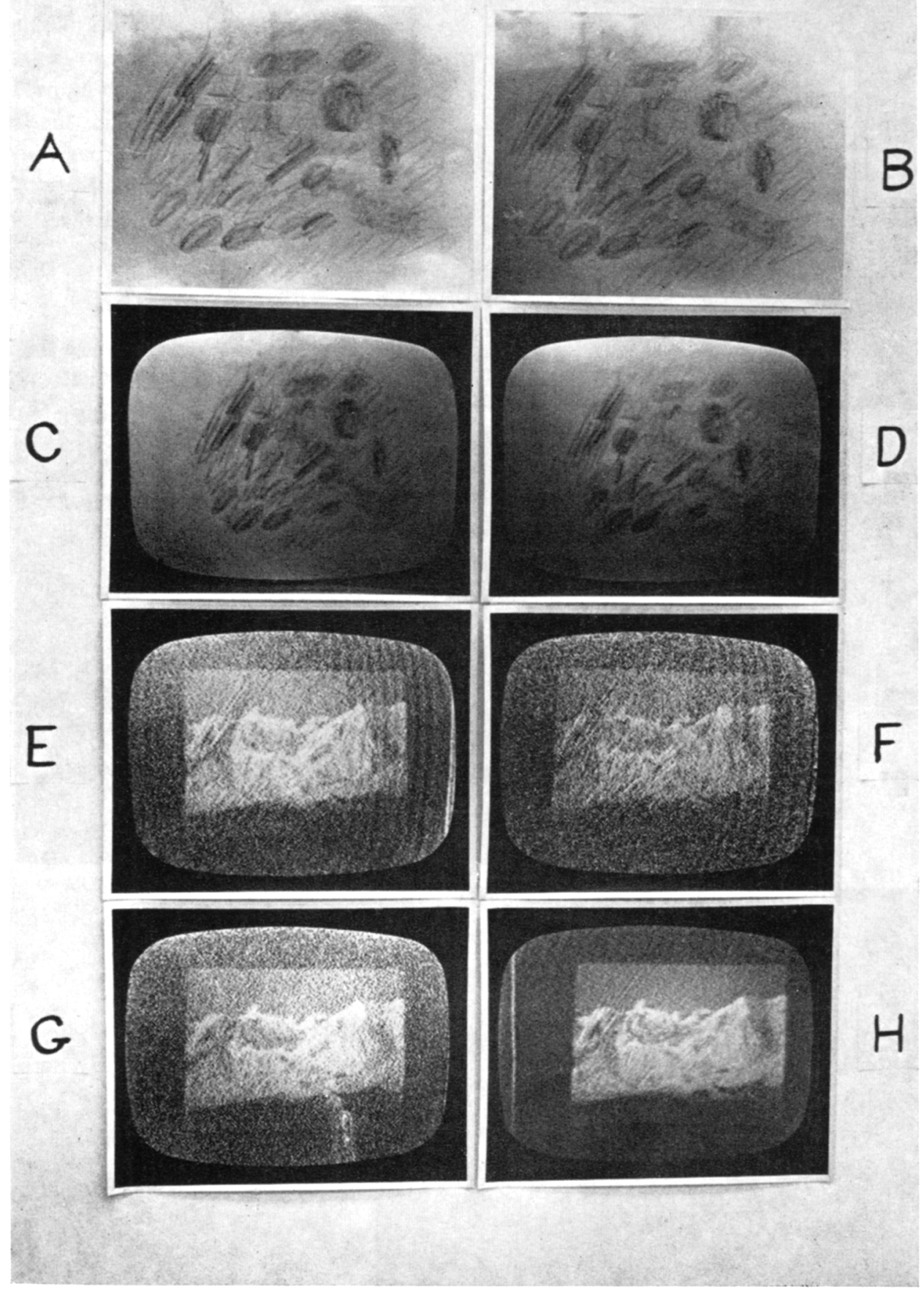

Fig. 5. A laboratory test of the system in the single camera mode. $A$ shows a test target. $B$ shows the same test target with a slide projected on it. Highlight intensity of the slide is about $2 \frac{1}{2} \%$ of the total light incident on the target. $C$ and $D$ show a television view of the target without and with the projected slide. $E$ and $F$ show the result of a simple single subtraction with a contrast enhancement of $10 . G$ is an average of $E$ and $F$. Notice the improvement in the signal-to-noise ratio and the cancelling out of systematic recording errors. $H$ shows an average of 128 subtracted pairs. The intensity of the sky in the mountain scene is about $0.1 \%$ of the total light intensity incident on the target. 


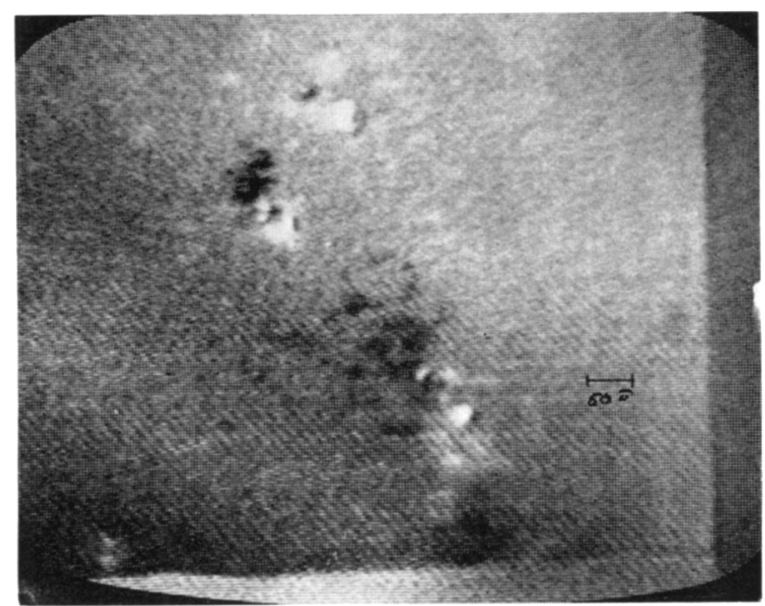

Fig. 6a.
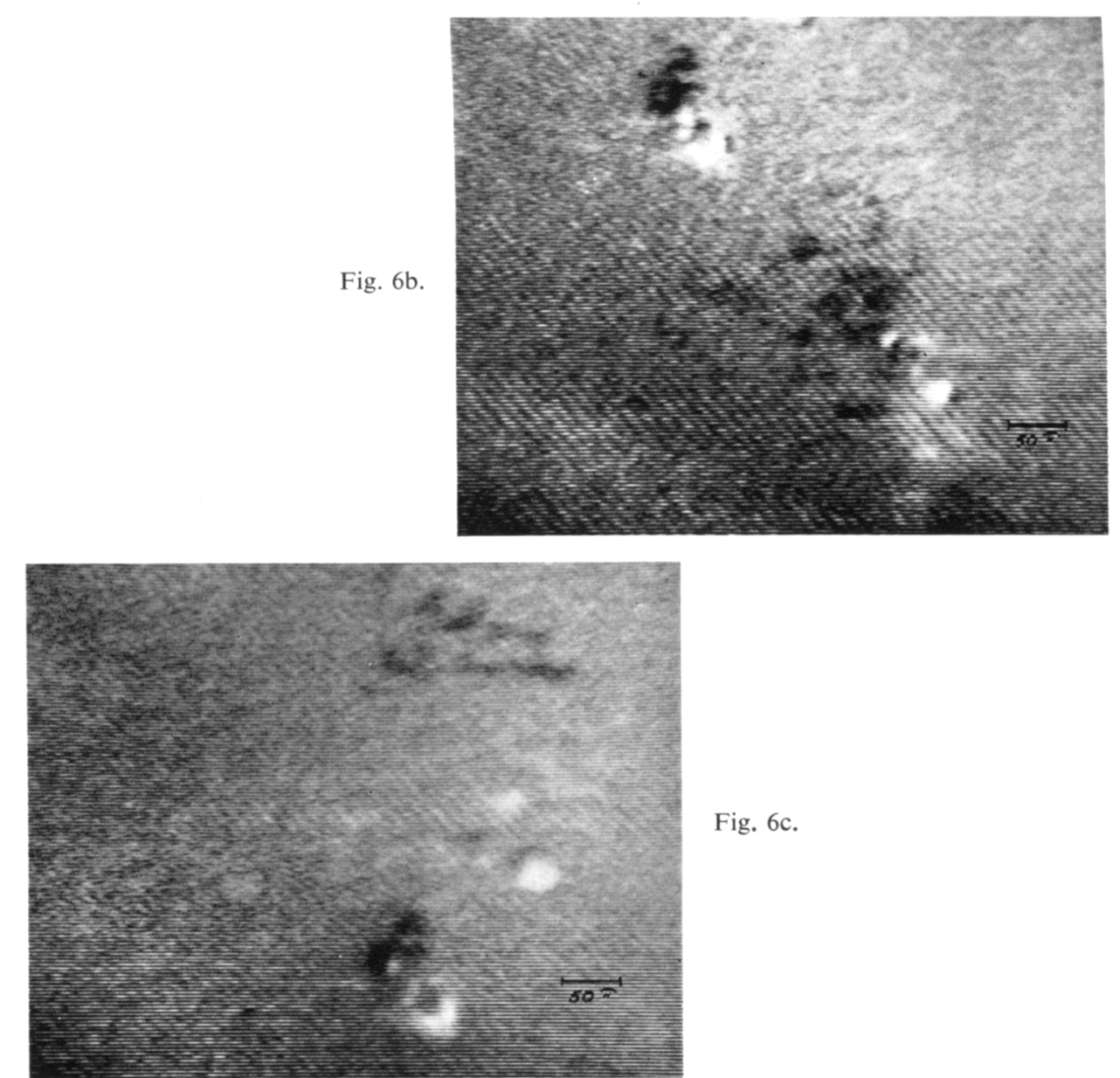

Fig. 6c.

Figs. 6a-c. Three videomagnetograms made at Caltech on October 17, 1970. The monochromatic images were made using a $\frac{1}{8} \AA$ bandpass Lyot filter kindly loaned to the authors by the Lockheed Solar Observatory. 
a manner similar to that used in the dual camera system. Figure 5 shows the results of a laboratory test of the system. Figure 6 shows some typical magnetograms. Both were taken using the photometer in the single-camera mode. As yet, little experience has been gained with the double-camera mode of operation. In the single-camera mode, about ten seconds are required to record the initial pictures and about $45 \mathrm{sec}$ to subtract and average them. Thus, about one magnetogram per minute can be made. The system is presently in use at Downs Laboratory at Caltech, in a study of magnetic fields in developing solar active regions.

\section{Acknowledgement}

This work was supported in part by the Office of Naval Research (ONR) under grant NONR-220(55) and the National Aeronautics and Space Administration (NASA) under grant NGR-05-002-142.

\section{References}

Leighton, R. B.: 1957, Astrophys. J. 130, 366.

Roberts, P. H.: 1970, Ph.D. Thesis, California Institute of Technology (unpublished). 